\title{
GASTOS E VOTOS NAS ELEIÇÕES 2012: O CASO DOS CANDIDATOS A PREFEITO DAS CAPITAIS BRASILEIRAS
}

\author{
Ranulfo Paranhos \\ Dalson Britto Figueiredo Filho \\ Enivaldo Carvalho da Rocha \\ José Alexandre da Silva Júnior \\ Antonio Lavareda*
}

Resumo: Analisa a relação entre receita de campanha e resultados eleitorais nas eleições municipais de 2012. Em termos substantivos, testou-se a hipótese de que desafiantes são mais eficientes do que incumbents (Efeito Jacobson). Metodologicamente, foi combinada a estatística descritiva e multivariada, além de um modelo regressão Poisson para analisar os dados disponibilizados pelo Tribunal Superior Eleitoral (TSE) referentes às disputas eleitorais ao cargo de prefeito em todas as capitais brasileiras. Os resultados sugerem que: (1) em média, o incremento de $1 \%$ na receita eleva em $0,646 \%$ o número de votos e (2) diferente do esperado, incumbents $(\beta=0,645 ; \mathrm{t}=3,785$ e $\mathrm{p}$-valor $<0,001)$ são mais eficientes do que os candidatos desafiantes $(\beta=0,607 ; \mathrm{t}=17,480$ e $\mathrm{p}$-valor $<0,000)$.

Palavras-chave: Receita de campanha. Resultados eleitorais. Eleição municipal (2012). Efeito Jacobson. Metodologia quantitativa.

\begin{abstract}
This paper analyzes the relationship between campaign spending and electoral outcomes in 2012 municipal elections. On substantive grounds, we test the hypothesis that challengers candidates are more efficient than incumbents (Jacobson's Effect). Methodologically, we combine descriptive and multivariate statistic with a

\footnotetext{
* Ranulfo Paranhos é Professor do Instituto de Ciências Sociais da Universidade Federal de Alagoas (ICS/UFAL), Doutorando e Mestre em Ciência Política pelo Departamento de Ciência Política da Universidade Federal de Pernambuco (DCP/UFPE) (ranulfoparanhos@me.com). Dalson Britto Figueiredo Filho é Professor do Departamento de Ciência Política da Universidade Federal de Pernambuco (DCP/UFPE), Doutor e Mestre em Ciência Política pelo Departamento de Ciência Política da Universidade Federal de Pernambuco (DCP/UFPE) (dalsonbritto@ yahoo.com.br). Enivaldo Carvalho da Rocha é Professor do Departamento de Ciência Política da Universidade Federal de Pernambuco (DCP/UFPE), Pós-doutorando do Departamento de Ciência Política da Universidade Federal de Minas Gerais (DCP/UFMG) e Mestre em Estatística pela Universidade de São Paulo (USP) e Doutor em Engenharia de Produção pela Universidade Federal do Rio de Janeiro (UFRJ) (enivaldocrocha@gmail.com). José Alexandre da Silva Júnior é Professor do Curso de Ciências Sociais da Universidade Federal de Goiás (UFG), Doutor e Mestre em Ciência Política pelo Departamento de Ciência Política da Universidade Federal de Pernambuco (DCP/UFPE) (jasjunior2007@yahoo.com.br). Antonio Lavareda é Professor do Departamento de Ciência Política da Universidade Federal de Pernambuco (DCP/UFPE), Mestre em Sociologia pela Universidade Federal de Pernambuco (UFPE) e Doutor em Ciência Política pelo Instituto Universitário de Pesquisas do Rio de Janeiro (IUPERJ)
} (lavareda@mci.com.br).
\end{abstract}


regression Poisson model to analyze data from Superior Electoral Court (TSE) regarding mayor electoral contests in all Brazilian state capitals. The results suggest that: (1) on average, an $1 \%$ increase in campaign spending produces a $0,646 \%$ increases in votes and (2) different from what we expected, incumbents $(\beta=0,645 ; \mathrm{t}=3,785 \mathrm{e}$ $\mathrm{p}$-value $<0,001)$ are more efficient than challengers candidates $((\beta=0,607 ; \mathrm{t}=17,480 \mathrm{e} p$-value $<0,000)$.

Keywords: Campaign spending. Electoral outcomes. Municipal elections (2012). Jacobson effect. Quantitative methodology.

Nihil tam munitum quod non expugnari pecunia possit (Nada é tão fortificado que não pode ser tomado com dinheiro)

Cícero

\section{Introdução}

Para Berry (1974, p. 120), “o financiamento das eleições continua sendo uma das áreas menos compreendidas do comportamento político Americano, [...] o dinheiro é certamente importante para explicar o sucesso eleitoral, mas quão importante e como é melhor utilizado continua sendo um grande mistério". Cerca de 40 anos depois desse alerta, o financiamento das eleições se tornou um dos temas mais estudados pela Ciência Política norte-americana. E o que explica essa transição? A oferta de dados. Depois da aprovação do Federal Election Campaign Act (1971) e suas emendas em 1974, diferentes inovações institucionais foram adotadas, entre elas, a criação da Federal Election Comission (FEC) e a obrigação de partidos e candidatos reportarem seus gastos de campanha. $\mathrm{O}$ efeito prático disso foi o desenvolvimento de uma subárea na Ciência Política que se preocupa em responder duas principais questões: (1) qual é o efeito dos gastos de campanha sobre os resultados eleitorais? e (2) qual é o efeito das contribuições de campanha sobre o comportamento congressual? (JACOBSON, 1985; SMITH, 2001, grifo do autor). Isso quer dizer que tanto o gasto quanto as contribuições de campanha são operacionalizados como variáveis independentes, tendo os resultados eleitorais e o comportamento congressual como variáveis dependentes, respectivamente.

Nesse trabalho, analisou-se a relação entre gastos de campanha e resultados eleitorais nas eleições municipais de 2012. Em termos substantivos, testou-se a hipótese de que desafiantes são mais eficientes do que incumbents (Efeito Jacobson). Metodologicamente, foi combinada a estatística descritiva, comparação de médias, regressão linear de mínimos quadrados ordinários (MQO) e um modelo de regressão Poisson para analisar os dados disponibilizados pelo Tribunal Superior Eleitoral (TSE) referentes às disputas eleitorais ao cargo de prefeito em todas as capitais brasileiras. 
Para proceder à investigação, esse trabalho está dividido em cinco seções. A próxima parte apresenta uma breve revisão da literatura empírica sobre gastos de campanha e resultados eleitorais. A terceira seção descreve as características do desenho de pesquisa. Depois disso, foram apresentados os resultados. Por fim, a quinta seção sumariza as principais conclusões.

\section{Revisão da Literatura}

Mensurar o efeito do gasto de campanha sobre os resultados eleitorais se tornou um tema canônico na Ciência Política (PALDA, 1973, 1975; WELCH, 1974, 1980; JACOBSON, 1978, 1985, 1990; GLANTZ, ABROMOWITZ e BURKHART, 1976; ABROMOWITZ, 1988, 1991; GREEN e KRASNO, 1988, 1990; GERBER, 1998, 2004) ${ }^{1}$. O desenho de pesquisa típico tem três principais características: (1) estima uma regressão da proporção de votos recebidos pelo candidato em função dos seus níveis de gasto, controlando por diferentes variáveis; (2) utiliza a forma funcional de mínimos quadrados ordinários ${ }^{2}$ e (3) tem como unidade de análise a Câmara dos Deputados dos Estados Unidos. De acordo com Gerber ${ }^{3}$ (2004), o modelo básico para analisar a relação entre gastos e votos é o seguinte:

$\operatorname{Votos}_{\text {inc }}=\alpha+\beta_{1} f\left(\right.$ gasto $\left._{\text {inc }}\right)+\beta_{2} f\left(\right.$ gasto $\left._{\text {chal }}\right)+\beta_{3} X+\varepsilon$

Em que $\operatorname{Votos}_{n c}$ representa a proporção de votos recebida pelo candidato incumbent, gasto $_{\text {inc }}$ representa o seu gasto total, gasto $_{\text {chal }}$ representa o gasto total do candidato desafiante (challenger) e $X$ representa variáveis adicionais que possivelmente influenciam os resultados eleitorais, como qualidade do candidato desafiante e características do eleitorado e/ou dos distritos eleitorais (GERBER, 2004) $)^{4}$.

Comparativamente, alguns trabalhos analisam as assembleias estaduais e o Senado (GRIER, 1989; GERBER, 1988). Em termos metodológicos, alguns especialistas utilizam modelos de mínimos quadrados em dois estágios (GREEN e KRASNO, 1988), transformações logarítmicas (JACOBSON, 1978), experimentos computacionais (HOUSER e STRATMANN, 2008) e experimentos de campo (GERBER, 2004) tentando identificar os mecanismos que conectam gastos

\footnotetext{
${ }^{1}$ Salvo melhor catalogação, Jacobson (1985) apresenta a primeira revisão da literatura empírica sobre a relação entre gastos de campanha e resultados eleitorais.

2 Especificamente no que diz respeito à forma funcional ver Welch (1974), Jacobson (1976) e Shepard (1977) para aplicações de modelos lineares. Welch (1976) para um modelo semi-log, Lott e Warner (1974) para um modelo log-log e Silberman e Yochum (1978) para um modelo quadrático.

${ }^{3}$ A constante do modelo é representada por $\alpha$ e os coeficientes de cada variável são representados pelos $\beta 1, \beta 2$ e $\beta 3$, respectivamente.

${ }^{4} \mathrm{O}$ termo incumbent é utilizado para designar o candidato que concorre à eleição enquanto possui um mandato, ou seja, tenta a reeleição. Os candidatos challengers (desafiantes) são aqueles que competem pela vaga do incumbent.
} 
e votos. Teoricamente, Jacobson produziu um dos trabalhos seminais na área (GERBER, 2004) ${ }^{5}$. A figura abaixo ilustra o que a literatura denominou de Efeito Jacobson.

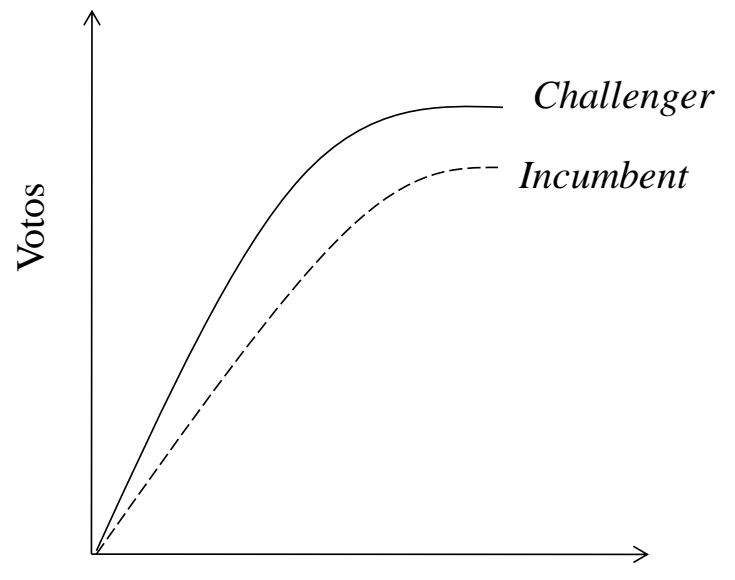

Gasto de campanha

Figura 1 - Efeito Jacobson

Fonte: Jacobson (1978).

Tanto o gasto de campanha dos desafiantes (challengers) quanto o dos incumbents exerce um efeito positivo sobre a quantidade de votos recebidos e sofrem de retornos marginais decrescentes. Ou seja, a partir de um determinado limite, cada unidade de dinheiro investida nas campanhas passa a ter um efeito progressivamente menor. No entanto, a inclinação da reta dos challengers é maior do que a dos incumbents, sugerindo que os candidatos desafiantes se beneficiam mais de cada unidade adicional de gasto em suas campanhas. Para Jacobson (1978), a principal implicação normativa desse fenômeno é o de que limitações na quantidade de dinheiro gasto nas campanhas tendem a favorecer o status quo, já que os desafiantes serão mais prejudicados. Em suas palavras, "qualquer regulamentação que reduza o nível de gasto dos candidatos beneficiará os incumbents. Isso inclui limitações nas contribuições de campanha de indivíduos e grupos, bem como tetos de gasto para os candidatos" (JACOBSON, 1978, p. 489). A tabela abaixo sintetiza parte dessa literatura.

\footnotetext{
${ }^{5}$ Gerber (2004) argumenta que "uma crítica recorrente aos achados de pesquisa de Jacobson é a de que os candidatos incumbents aumentam os seus níveis de gasto de acordo com o nível de competição política. Se as variáveis de controle não forem devidamente especificadas, os efeitos do gasto de campanha tendem a ser sub-estimados dada a correlação negativa entre o gasto de campanha do incumbent e o termo de erro do modelo de regressão" (GERBER, 2004, p. 542). Isso quer dizer que os coeficientes negativos encontrados por Jacobson em relação ao gasto de campanha dos incumbents podem ser explicados por um erro de especificação do modelo na medida em que ele não incluiu uma variável para controlar pela qualidade dos candidatos desafiantes (challengers).
} 
Tabela 1 - Síntese da literatura

\begin{tabular}{l|l}
\hline \hline \multicolumn{1}{c|}{ Autor (ano) } & \multicolumn{1}{c}{ Argumento } \\
\hline $\begin{array}{l}\text { Jacobson (1978); Abromowitz, } \\
\text { (1988); Ansolabehere e Gerber, } \\
\text { (1994); Gerber (2004) }\end{array}$ & $\begin{array}{l}\text { O gasto do incumbent é ineficiente, mas o gasto do challenger } \\
\text { produz vários ganhos (o coeficiente de regressão associado ao } \\
\text { gasto de campanha do challenger é positivo e estatisticamente } \\
\text { significativo) }\end{array}$ \\
\hline $\begin{array}{l}\text { Erikson e Palfrey (2000); Green } \\
\text { e Krasno (1988); Gerber } \\
\text { (1998); Levitt (1994) }\end{array}$ & $\begin{array}{l}\text { Após controlar por outras variáveis, nem o gasto do incumbent } \\
\text { nem o gasto do challenger exercem efeitos expressivos (não há } \\
\text { diferenças significativas no efeito do gasto para os dois grupos) }\end{array}$ \\
\hline $\begin{array}{l}\text { Thomas (1989); Kenny e } \\
\text { McBurnett (1994); Goidel e } \\
\text { (2001) }\end{array}$ & $\begin{array}{l}\text { Após controlar pela qualidade do challenger e por causalidade } \\
\text { recursiva, o efeito marginal do gasto do incumbent é substancial } \\
\text { (o coeficiente de regressão é positivo e estatisticamente } \\
\text { significativo) }\end{array}$ \\
\hline $\begin{array}{l}\text { Krasno, Green e Cowden } \\
(1994)\end{array}$ & $\begin{array}{l}\text { O gasto de campanha do incumbent é dependente (reativo) do } \\
\text { gasto do challenger }\end{array}$ \\
\hline \hline
\end{tabular}

Fonte: Elaboração dos autores

Levitt (1994, p. 777) argumenta que "o gasto de campanha exerce um impacto extremamente pequeno sobre os resultados eleitorais, independente do tipo de candidato". Gerber (1998, p. 401) defende que "quando a endogeneidade dos níveis de gasto é propriamente controlada, os efeitos marginais do gasto de incumbents e desafiantes são aproximadamente iguais". Para Jacobson (1990, p. 470),

os modelos de regressão linear de mínimos quadrados ordinários utilizados na maior parte dos estudos são inapropriados para estimar relações recíprocas; é necessário utilizar um sistema de equações simultâneas. As estimativas dos parâmetros utilizando a forma funcional de mínimos quadrados quando a verdadeira relação é recíproca são enviesadas e inconsistentes.

Existe discordância, também, entre os estudos que empregam modelos de mínimos quadrados em dois estágios, tentando corrigir o problema da endogeneidade. Por exemplo, Green e Krasno (1988) encontram coeficientes de regressão positivos e estatisticamente significativos para os incumbents. Por outro lado, Jacobson (1978, p. 475) argumenta que "o gasto dos candidatos desafiantes tem um efeito substancial sobre o resultado eleitoral quando o problema da simultaneidade é eliminado da equação". 
No Brasil, a literatura sobre financiamento de campanha e resultados eleitorais tem se desenvolvido progressivamente ${ }^{6}$. Por exemplo, Portugal e Bugarin (2003) modelaram o efeito do financiamento público das campanhas eleitorais sobre bem-estar social e representação no legislativo. Peixoto (2008) analisou o efeito dos gastos de campanha sobre a votação de deputados estaduais e federais em 2002 e em 2006. Cervi (2009) examinou a relação entre instituições democráticas e o financiamento de campanha. Similarmente, Campos (2009) analisou os custos do sistema eleitoral no Brasil. Mais recentemente, Lemos, Marcelino e Pederiva (2010) estimaram o impacto dos gastos sobre os resultados eleitorais de deputados federais e senadores nas eleições de 2002 e 2006. Bruno Speck tem contribuído não só teoricamente, mas também em nível organizacional na medida em que articulou um grupo de estudo sobre o financiamento das campanhas no Brasil. Sua produção inclui trabalhos sobre a relação entre financiamento de campanha e corrupção, bem como artigos em perspectiva comparada ${ }^{7}$.

\section{Metodologia $^{8}$}

Com o objetivo de maximizar a replicabilidade do estudo (KING, 1995), essa seção descreve as características do desenho de pesquisa, enfatizando o processo de coleta e elaboração do banco de dados ${ }^{9}$. O quadro abaixo sumariza as principais características do nosso desenho de pesquisa.

\footnotetext{
${ }^{6}$ Para o estudo pioneiro sobre o Brasil, ver os trabalhos de David Samuels (20001a, 2001b, 2001c).

${ }^{7}$ Para um trabalho sobre o financiamento de campanha em perspectiva comparada ver Scarrow (2007).

${ }^{8}$ Os dados utilizados nesse trabalho estão disponíveis no seguinte endereço eletrônico: http://www.tse.jus.br/eleicoes/repositorio-de-dados-eleitorais

9 Para King, Keohane e Verba (1994), "all data and analyses should, insofar as possible, be replicable. Replicability applies not only to the data, so that we can see whether our measures are reliable, but to the entire reasoning process used in producing conclusions" (KING, KEOHANE e VERBA 1994, p. 26).
} 
Quadro 1 - Desenho de pesquisa

\begin{tabular}{|c|c|}
\hline População ${ }^{10}$ & Candidatos ao cargo de prefeito das capitais brasileiras em 2012 \\
\hline Variáveis & $\begin{array}{l}\text { VD: número de votos } \\
\text { VI: Receita de campanha }\end{array}$ \\
\hline Hipóteses & $\begin{array}{l}\text { Candidatos desafiantes são mais eficientes em transformar receita em } \\
\text { votos }^{11} \\
\text { (Efeito Jacobson) }\end{array}$ \\
\hline Técnicas & $\begin{array}{l}\text { Estatística descritiva, comparação de médias, análise de variância } \\
\text { (ANOVA), regressão de mínimos quadrados ordinários (MQO) e } \\
\text { regressão Poisson. }\end{array}$ \\
\hline
\end{tabular}

Fonte: Elaboração dos autores.

A população de interesse é composta pelos candidatos ao cargo de prefeito das capitais brasileiras nas eleições de 2012. A variável dependente é o número de votos nominais. A variável explicativa é a quantidade de recursos investida na campanha (Receita). Em termos substantivos, testaremos a hipótese de que o efeito do gasto é maior para os candidatos desafiantes em relação aos incumbents (Efeito Jacobson). Tecnicamente, utilizamos estatística descritiva, teste $t$ de comparação de médias, análise de variância (ANOVA), regressão linear de mínimos quadrados ordinários (MQO) e um modelo de regressão Poisson.

\section{Resultados e Discussões}

Nessa seção apresentamos dois conjuntos de informações: (1) estatística descritiva da variável independente (Receita) e (2) as estimativas do impacto da receita sobre os votos, tanto no nível agregado (todos os candidatos juntos) quanto por tipo de candidato (teste de hipótese). A tabela abaixo sumariza a estatística descritiva dos gastos de campanha dos candidatos ao cargo de prefeito das capitais brasileiras nas eleições de 2012.

\footnotetext{
${ }^{10}$ Tecnicamente, o ideal seria utilizar uma amostra aleatória simples para selecionar os casos. No entanto, o esforço de compatibilização dos bancos de dados seria proibitivo. Para explicar, o TSE disponibiliza RECEITA e VOTOS em bancos de dados distintos e de forma desagregada. Dessa forma, o primeiro passo da coleta consiste em agregar todas as receitas recebidas por um determinado candidato, computando o total. O mesmo procedimento deve ser repetido para o número de votos. Depois de produzir os bancos agregados, é necessário unir esses bancos. Além disso, muitos candidatos tem votos mas não reportaram receitas, o que minimiza as chances de compatibilização automática. Optamos por iniciar pelas capitais, depois coletar os dados referentes aos municípios com mais de 200 mil habitantes e finalizar com os municípios menores.

${ }^{11}$ Tecnicamente, isso quer dizer que o coeficiente de regressão não padronizado dos incumbents ( $\beta$ i) deve ser maior do que o dos candidatos desafiantes $(\beta c)$. A principal consequência disso é que o teste de hipótese poderia ser unicaudal já que assumimos que $\beta \mathrm{i}>\beta \mathrm{c}$. Contrariamente, em uma hipótese que sustentasse apenas a diferença entre os coeficientes $(\beta i \neq \beta c), \beta i$ poderia ser maior ou menor do que $\beta c$. O teste unicaudal permite que a hipótese nula seja mais facilmente rejeitada em comparação com o teste bi-caudal. Como o teste bi-caudal é mais conservador, iremos sempre optar por ele.
} 
Tabela 2 - Estatística descritiva da receita de campanha (2012)

\begin{tabular}{c|c|c|c|c|c}
\hline $\mathbf{N}$ & mínimo & máximo & média & mediana & desvio padrão \\
\hline 180 & 500,00 & $40.523 .673,00$ & $2.627 .985,50$ & $398.002,00$ & $5.268 .806,05$ \\
\hline
\end{tabular}

Fonte: Elaboração dos autores.

Em média, uma campanha custa $\mathrm{R} \$ 2.627 .985,50$, com um erro padrão de 392.713,62 e um desvio padrão de 5.268.806,05. A magnitude do desvio em relação à média sugere que a distribuição da receita é fortemente assimétrica. O histograma abaixo ilustra a distribuição da receita.

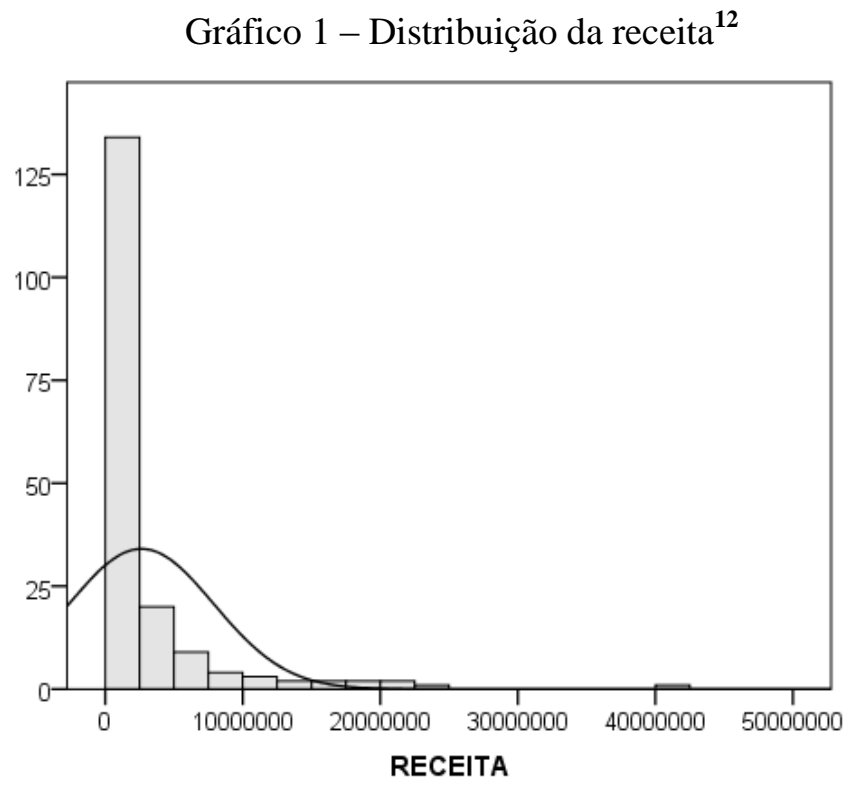

Fonte: Elaboração dos autores.

O próximo passo é analisar como a receita varia por tipo de candidato. O gráfico abaixo ilustra essas informações.

\footnotetext{
${ }^{12}$ Nos anexos reportamos um boxplot da receita e um boxplot por Estado.
} 
Gráfico 2 - Receita de campanha por tipo de candidato (IC 95\%)

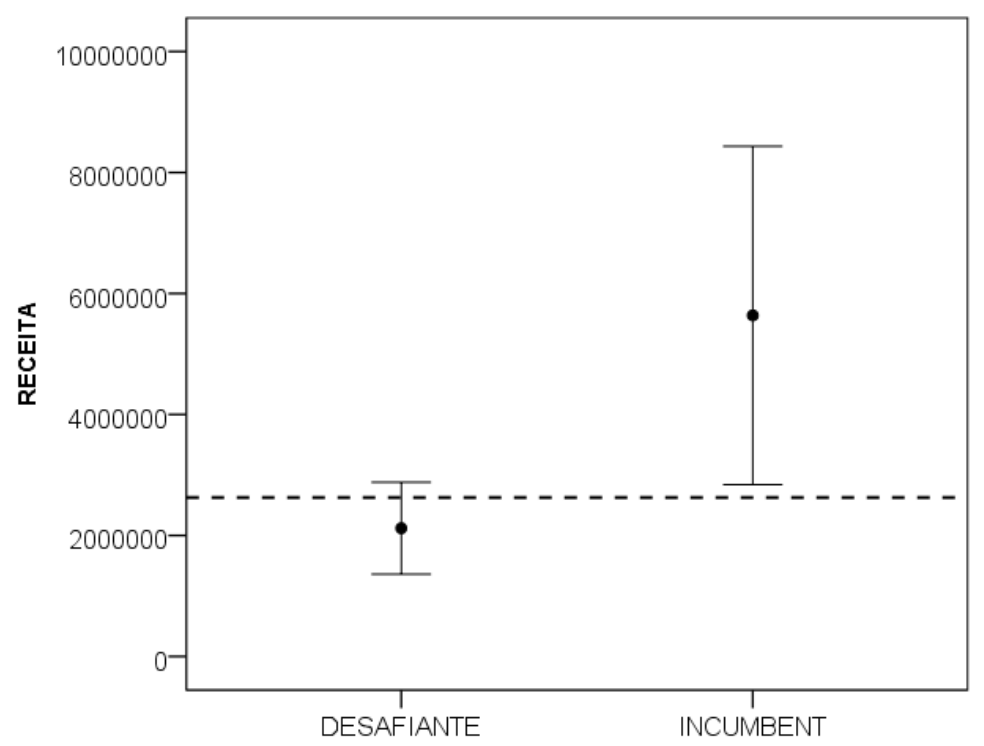

Fonte: Elaboração dos autores.

A linha pontilhada representa a média geral ( $\mathrm{R} \$ 2.627 .985,50)$. Comparativamente, incumbents investem mais recursos em suas campanhas do que candidatos desafiantes (dif = 3.517.407,22) $)^{13}$. Enquanto um incumbent típico gasta cerca de $\mathrm{R} \$ 5.637 .322,77$, o desafiante investe aproximadamente $\mathrm{R} \$ 2.119 .915,55$. A tabela abaixo sumariza a estatística descritiva por tipo de candidato.

Tabela 3 - Estatística descritiva por tipo de candidato

\begin{tabular}{c|c|c|c|c}
\hline $\begin{array}{c}\text { Tipo de } \\
\text { Candidato }\end{array}$ & N & média & desvio padrão & $\begin{array}{c}\text { coeficiente de } \\
\text { variação }\end{array}$ \\
\hline Desafiante & 154 & $2.119 .915,55$ & $4.779 .076,64$ & 2,25 \\
\hline Incumbent & 26 & $5.637 .322,77$ & $6.926 .558,61$ & 1,23 \\
\hline
\end{tabular}

Fonte: Elaboração dos autores.

\footnotetext{
${ }^{13}$ Como qualquer teste paramétrico, o teste $\mathrm{t}$ de comparação de médias deve ser realizado assumindo distribuição normal das variáveis. No entanto, a partir do Teorema Central do Limite, sabemos que quando o tamanho da amostra aumenta, a distribuição amostral de sua média se aproxima cada vez mais de uma distribuição normal. Outro elemento que deve ser observado é a homogeneidade da variância entre os grupos comparados. Aqui, os resultados sugerem um $\mathrm{F}=9,235$ com p-valor de 0,003 , sugerindo que esse pressuposto foi violado. A comparação deve ser então realizada assumindo que a variância entre os grupos é diferente $(\mathrm{t}=2,491 \mathrm{e}$-valor $=0,019)$.

${ }^{14} \mathrm{O}$ coeficiente de variação é uma medida de dispersão para comparar médias de distribuições diferentes. Isso porque o desvio padrão é relativo à média e como em duas distribuições as médias podem ser diferentes, o desvio dessas duas distribuições pode não ser diretamente comparável. Uma solução é utilizar o coeficiente de variação: basta dividir o valor do desvio-padrão pela média
} 
Depois de analisar o padrão de gasto dos candidatos ao cargo de prefeitos das capitais brasileiras em 2012, é importante examinar o nível de associação entre receita e votos. O gráfico de dispersão abaixo ilustra a correlação linear entre essas duas variáveis.
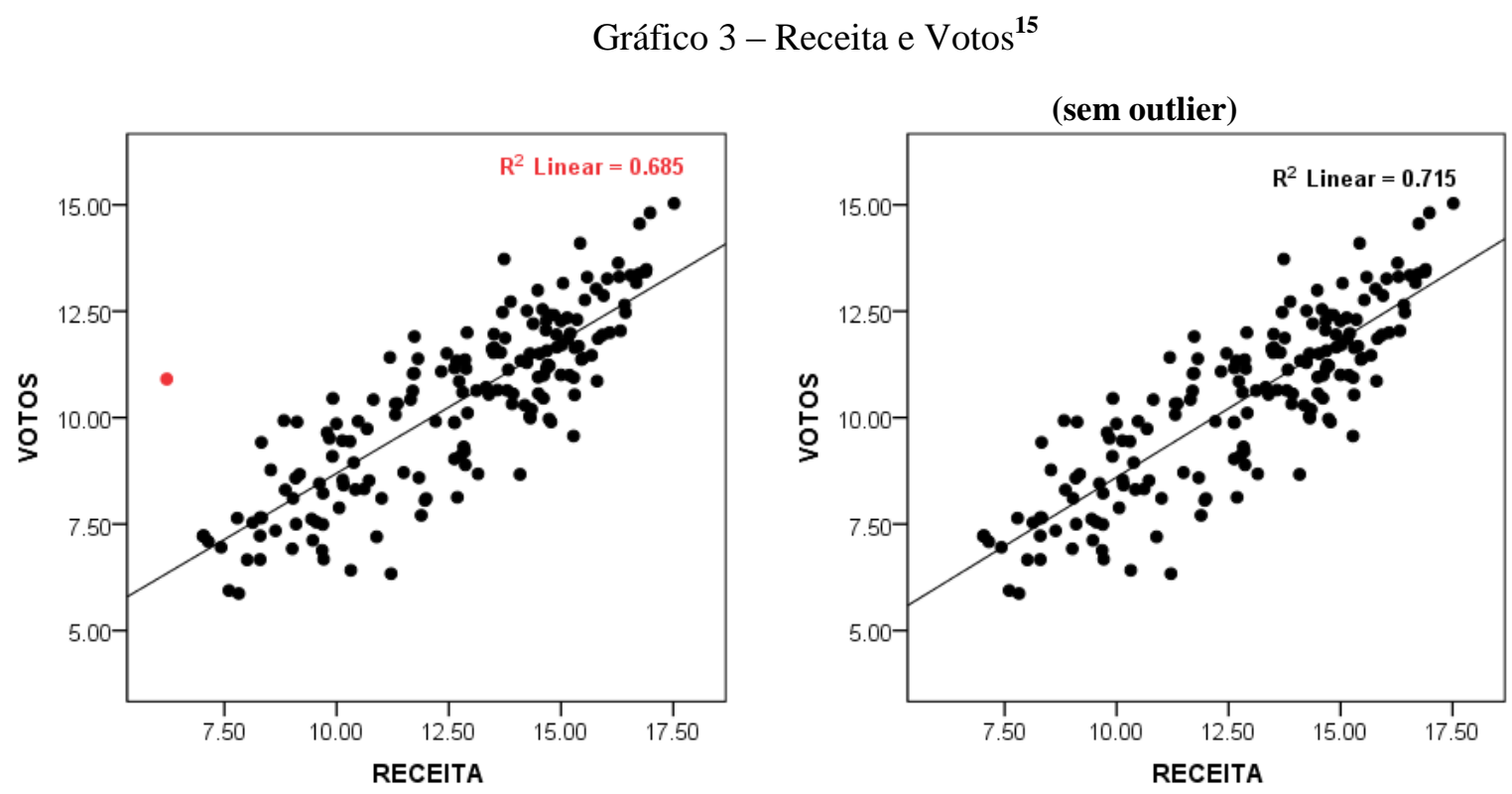

Fonte: Elaboração dos autores.

Observa-se uma correlação positiva $(0,846$; p-valor $<0,001$ e $n=174)$ entre receita de campanha $(x)$ e número de votos $(y)$. O coeficiente de determinação $\left(\mathrm{r}^{2}\right)$ é de 0,685 , considerando-se todos os casos. Ou seja, em média, a variação da receita explica $68,5 \%$ da variação do número de votos. No entanto, sem a presença do outlier (ponto vermelho) ${ }^{16}$, o ajuste do modelo melhora sensivelmente $\left(\mathrm{r}^{2}=0,715\right)$. A tabela abaixo sumariza as estimativas do modelo linear de mínimos quadrados ordinários.

Tabela 4 - Coeficientes

\begin{tabular}{|c|c|c|c|c|c|}
\hline \multirow[t]{2}{*}{ Modelo MQO } & \multicolumn{2}{|c|}{$\begin{array}{c}\text { Coeficientes não } \\
\text { padronizados }\end{array}$} & \multirow{2}{*}{$\begin{array}{c}\text { Coeficientes } \\
\text { padronizados }\end{array}$} & \multirow[t]{2}{*}{$\mathrm{t}$} & \multirow[t]{2}{*}{ Sig. } \\
\hline & $\mathrm{B}$ & Erro padrão & & & \\
\hline (Constante) & 2,143 & 0,403 & & 5,314 & 0,000 \\
\hline Receita & 0,646 & 0,031 & 0,846 & 20,778 & 0,000 \\
\hline
\end{tabular}

Fonte: Elaboração dos autores.

Em média, o incremento de $1 \%$ da receita de campanha produz uma elevação de $0,646 \%$ na quantidade de votos $(\mathrm{t}=20,778$ e p-valor $<0,000)$. O próximo passo é testar a hipótese do trabalho.

\footnotetext{
${ }^{15}$ Ambas as variáveis foram transformadas utilizando o logarítimo neperiano.

${ }^{16}$ Maria da Consolação Rocha (PSOL - MG), teve uma receita declarada de R \$ 500 e obteve 54.530 votos.
} 
O gráfico abaixo ilustra a correlação entre receita de campanha e votos por tipo do candidato (incumbents e desafiantes).

Gráfico 4 - Gastos e votos por tipo de candidato

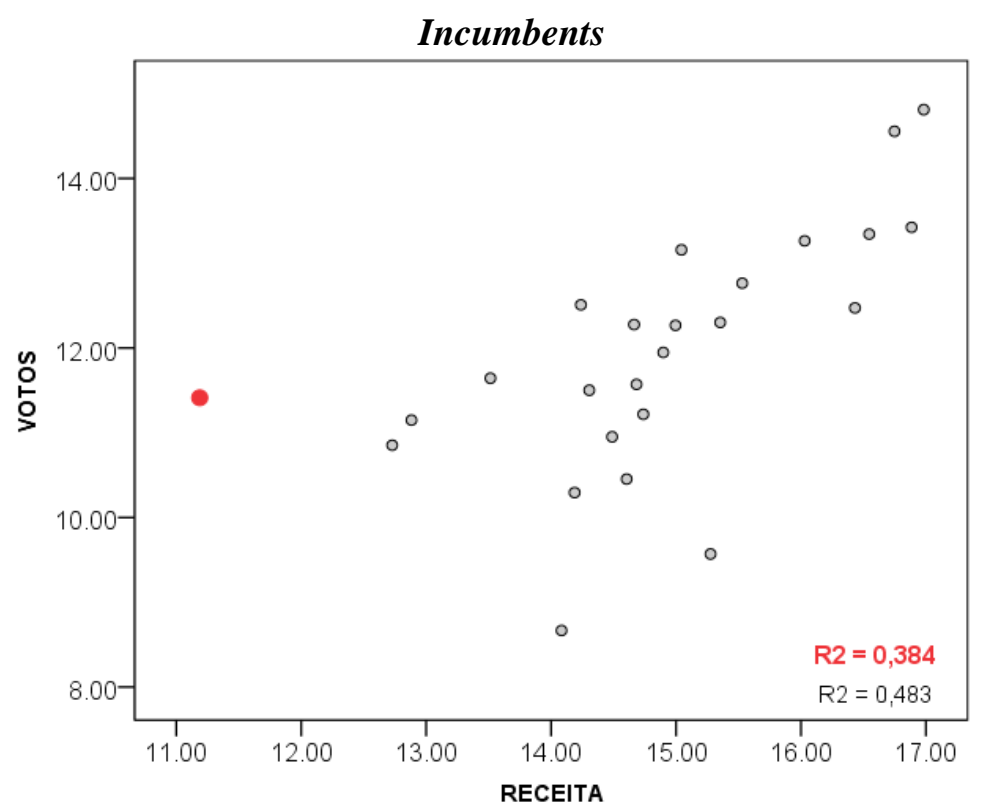

Fonte: Elaboração dos autores.

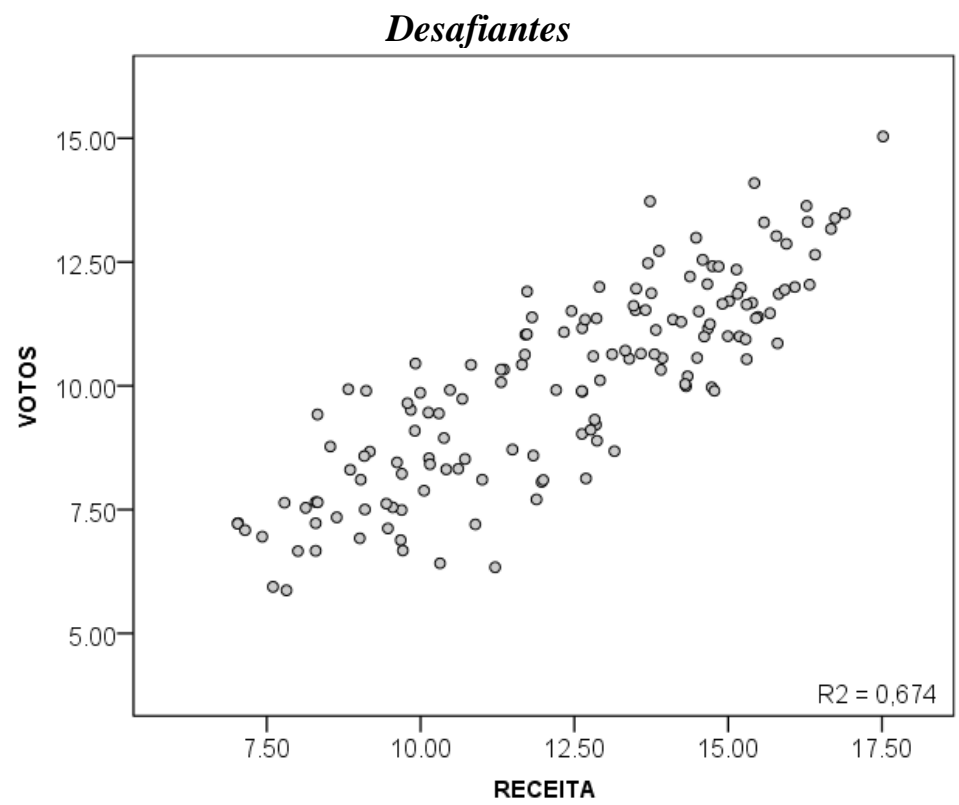

Fonte: Elaboração dos autores. 
Ao se desagregar a análise por tipo de candidato, observa-se que o coeficiente de determinação $\left(\mathrm{r}^{2}\right)$ dos incumbents é de 0,384 , considerando o outlier ${ }^{17}$. Sem ele, a variância da quantidade de votos explicada pela variância da receita passa para 48,3\%. Para os candidatos desafiantes, o $\mathrm{r}^{2}$ é de 0,674 . No entanto, ao se considerar o impacto, medido pelo coeficiente de regressão não padronizado, os resultados sugerem que desafiantes $(\beta=0,607 ; \mathrm{t}=17,48$ e pvalor $<0,000)$ são menos eficientes do que incumbents $(\beta=0,866 ; \mathrm{t}=4,535$ e $\mathrm{p}$-valor $<0,000)$ em transformar receita em votos. Em particular, a cada $1 \%$ no incremento da receita dos candidatos da situação, espera-se uma elevação, média, de 0,866\% na quantidade de votos recebidos, em comparação a $0,607 \%$ para os desafiantes ${ }^{18}$. Esses resultados contrariam a hipótese de trabalho, ou seja, o efeito Jacobson não se aplica às eleições majoritárias municipais nas capitais brasileiras em 2012.

Para garantir resultados mais robustos, estimamos um modelo de regressão Poisson, tendo como variável dependente o número de votos e como variável independente a receita de campanha. A tabela abaixo sumariza os coeficientes.

Tabela 5 - Coeficientes

\begin{tabular}{c|c|c|c|c|c|c}
\hline \hline \multirow{2}{*}{ Modelo Poisson } & \multicolumn{2}{|c|}{ Coeficientes } & & \multirow{2}{*}{ Sig. } & \multirow{2}{*}{ Exp (B) } \\
\cline { 3 - 6 } & B & Erro padrão & Wald $\chi^{2}$ & & \\
\hline & (Constante) & 0,447 & 0,000 & $36.735,33$ & 0,000 & 1,564 \\
\cline { 2 - 7 } & Receita & 0,793 & 0,000 & $28.438 .767,92$ & 0,000 & 2,209 \\
\hline \hline
\end{tabular}

Fonte: Elaboração dos autores.

O modelo Poisson corrobora os achados do modelo linear de mínimos quadrados ordinários. A receita exerce um efeito positivo e estatisticamente significativo sobre a quantidade de $\operatorname{votos}(\mathrm{B}=0,793 ; \operatorname{Exp}(\mathrm{B})=2,209 ; \mathrm{p}$-valor $<0,000)$. Comparativamente, incumbents $(\operatorname{Exp}(\mathrm{B})=$ $2,804)$ se beneficiam mais de cada real investido do que candidatos desafiantes $(\operatorname{Exp}(B)=2,041)$.

\section{Conclusão}

Esse trabalho analisou a relação entre receita de campanha e resultados eleitorais nas eleições municipais de 2012. Em particular, testamos a hipótese de que candidatos desafiantes são mais eficientes do que incumbents em transformar recursos em votos (Efeito Jacobson). Todavia, diferente de nossa expectativa inicial, os resultados sugerem o contrário, ou seja, incumbents são mais eficientes do que os candidatos desafiantes.

\footnotetext{
${ }^{17}$ Marcus Alexandre Medici Aguiar (PT - AC), teve uma receita declarada de R\$ 72.200 e obteve 90.557 votos.

${ }^{18}$ Outra forma de avaliar essa relação é replicar o modelo básico proposto por Gerber (2004) e incluir uma variável dicotômica para diferenciar candidatos incumbents e desafiantes, além de suas receitas de campanha. O coeficiente associado a incumbency foi de 0,271, sugerindo que um candidato da situação recebe, em média, 27,10\% a mais de votos do que um candidato desafiante, controlando pela receita.
} 
Dada a limitação da amostra (apenas capitais) e a restrição temporal (apenas as eleições de 2012), os resultados devem ser observados com bastante cautela. Além disso, a especificidade do cargo de prefeito não permite que façamos inferências válidas para outros cargos executivos, nomeadamente governador e presidente. É necessário que outros estudos coloquem nossos achados a teste no sentido de estimar mais acuradamente que tipo de candidato é, de fato, mais eficiente. Por outro lado, se o efeito anti-Jacobson for observado em outras amostras e em outras eleições, estaremos confiantes de que fizemos uma contribuição importante na medida em que conseguimos identificar um novo efeito. Resta agora aperfeiçoar o desenho de pesquisa no sentido de desvendar os mecanismos causais responsáveis por esse fenômeno.

Em termos metodológicos, adotamos um modelo de artigo mais enxuto, prezando pela clareza dos objetivos, métodos e resultados com o objetivo de elaborar um produto mais rapidamente consumível. Acreditamos fortemente que esse modelo pode contribuir positivamente para o desenvolvimento do conhecimento acumulado em Ciência Política ao enfatizar a objetividade e, principalmente, a replicabilidade dos resultados.

\section{Referências}

ABRAMOWITZ, A. Incumbency, campaign spending, and the decline of competition in U. S. house elections. Journal of Politics, West Nyack, v. 53, p. 34-56, February, 1991.

ABRAMOVITZ, A. Explaining Senate Election Outcomes. American Political Science Review, Washington-DC, v. 82, v. 2, p. 385-403, May, 1988.

BERRY, D. Electoral economics: Getting and spending. Polity, Palo Alto, 7, p. 118-139, may, 1974.

CAMPOS, M. M. Democracia, partidos e eleições: os custos do sistema partidário-eleitoral no Brasil. 2009. Tese (Doutorado em Ciência Política) - Faculdade de Filosofia e Ciências Humanas, Universidade Federal de Minas Gerais, Belo Horizonte, 2009.

CERVI, E. U. Instituições democráticas e financiamento de campanhas no Brasil: análises das contribuições de pessoas físicas, jurídicas e partidos políticos às eleições de 2008 e o financiamento público exclusivo de campanha. In: ENCONTRO ANUAL DA ASSOCIAÇÃO NACIONAL DE PÓS-GRADUAÇÃO EM CIÊNCIAS SOCIAIS, 33., 2009, Caxambu, MG. Anais... Caxambu: Editora, 2009. p.1-30.

GERBER, A. Does Campaign Spending Work?: Field Experiments Provide Evidence and Suggest New Theory. American Behavioral Scientist, Thousand Oaks, v. 47, n. 5, p. 541-574, February, 2004.

GERBER, A. Estimating the Effect of Campaign Spending on Senate Election Outcomes Using Instrumental Variables. American Political Science Review, Washington-DC, v. 92, n. 2, September, 1998.

GLANTZ, S. A.; ABRAMOWITZ, A. I.; BURKART M. P. Election Outcomes: Whose Money Matters? Journal of Politics, West Nyack, v. 38, p. 1033-1038, November 1976. 
GREEN, D. P.; KRASNO, J. S. Rebuttal to Jacobson's "New Evidence for Old Arguments". American Journal of Political Science, Hoboken-NJ, v. 34, n. 2, p. 363-372, May 1990.

GREEN, D. P.; KRASNO, J. S. Salvation for the Spendthrift Incumbent: Reestimating the Effects of Campaign Spending in House Elections. American Journal of Political Science, Hoboken-NJ, v. 32, p. 884-907, October, 1988.

GRIER, K. Campaign spending and senate elections, 1978-1984. Public Choice, Ney York, v. 63, n. 3, p. 201-220, December, 1989.

HOUSER, D.; STRATMANN, T. Selling Favors in the Lab: experiments on Campaign Finance Reform, Public Choice, New York, v. 136, p. 215-239, April 2008.

JACOBSON, G. C. The Effect of Campaign Spending in Election Octomes: New Evidences for Old Arguments. American Journal of Political Science, Hoboken-NJ, v. 34, p. 334-362, May 1990.

JACOBSON, G. C. Money and votes reconsidered: Congressional elections, 1972-1982. Public Choice, Ney York, v. 47, p. 7-62, January, 1985.

JACOBSON, G. C. The Effect of Campaign Spending in Congressional Elections. American Political Science Review, Washington-DC, v. 72, p. 469-491, May, 1978.

JACOBSON, G. C. Public funds for congressional candidates: who would benefit? Public Policy, Ney York v. 24, p. 1-32, February, 1976.

LOTT, W. F.; WARNER, P. D. The relative importance of campaign expenditures: An application of production theory. Quality \& Quantity, v. 8, Thousand Oaks, p. 99-106, February, 1974.

KING, G.; KEOHANE, R; VERBA, S. Designing Social Inquiry. New Jersey: Princeton University Press, 1994.

LEMOS, L. B., MARCELINO, D.; PEDERIVA, J. H. Porque dinheiro importa: a dinâmica das contribuições eleitorais para o Congresso Nacional em 2002 e 2006. Opin. Publica, Campinas, v.16, n. 2, p. 366-393, Novembro, 2010.

LEVITT, S. D. Using Repeat Challengers to Estimate the Effect of Campaign Spending on Election Outcomes in the U.S. House. Journal of Political Economy, Chicago, v. 102, n. 4, p. 777-798, Aug. 1994.

PEIXOTO, V. Impacto dos gastos de campanhas nas eleições de 2006. In: ENCONTRO ANUAL DA ASSOCIAÇÃO NACIONAL DE PÓS-GRADUAÇÃO EM CIÊNCIAS SOCIAIS, 32., 2008, Caxambu, MG. Anais... Caxambu, 2008. p.1-58.

PALDA, K. Does Advertising Influence Votes? An Analysis of the 1966 and 1970 Quebec Elections. Canadian Journal of Political Science, Ottawa, v. 6, December, 1973.

PALDA, K. The Effect of Expenditure on Political Success. Journal of Law and Economics, Chicago, v. 18, n. 3, p. 745-771, Dec. 1975.

PORTUGAL, A. C.; BUGARIN, M. Financiamento público e privado de campanhas eleitorais: efeitos sobre bem-estar social e representação partidária no Legislativo. Revista de Economia Aplicada, Ribeirão Preto-SP, (7) 3: 549-584, Outubro, 2003.

SAMUELS, D. Does Money Matter? Campaign Finance in Newly Democratic Countries: Theory and Evidence from Brazil. Comparative Politics, New York, v. 34, p. 23-42, October, 2001a.

SAMUELS, D. Incumbents and Challengers on a level Playing Field: Assessing the impact of Campaign Finance in Brazil. The Journal of Politics, West Nyack, v. 63, n. 2, p. 569-584, November, $2001 b$. 
SAMUELS, D. Money, Elections and Democracy in Brazil. Latin American Politics and Society, Hoboken, v. 43, n. 7, p. 27-48, July, 2001c.

SCARROW, S. Political Finance in Comparative Perspective. Annual Review of Political Science. Palo Alto, v. 10, 193-210. June, 2007.

SHEPARD, L. Does Campaign Spending Really Matter?. The Public Opinion Quarterly, Oxford, v. 41, p. 196-205, Summer, 1977.

SILBERMAN, J.; YOCHUM, G. The Role of Money in Determining Election Outcomes. Social Science Quarterly, Hoboken, v. 58, p. 671-682, November, 1978.

SMITH, B. A. Unfree Speech: the Folly of Campaign Finance Reform. Princeton, Princeton University Press, 2001.

SPECK, B. W. A compra de votos: uma aproximação empírica. Opinião Pública, Campinas, v. 9, n. 1, 2003. p. 148-169.

WELCH, W. The Allocation of Political Monies: Economic Interest Groups. Public Choice, New York, v. 35, p. 97-120, February,1980.

WELCH, W. (1974). The Economics of Campaign Funds. Public Choice, Ney York, v. 20, p. 8397, Winter 1974.

Artigo recebido em: 07/03/2013

Artigo aceito para publicação em: 20/05/2013 